adipocytokines were significantly higher in PsA compared to HD. The body mass index values were significantly correlated with the clinical inflammatory parameters (CRP and ESR) and activity of the disease (swollen joints count and DAS28). Increased levels of HOMA-IR also correlated with DAS28, clinical and serological inflammatory markers, and diverse adipokines. Elevated levels of cytokines correlated with the activity of the disease and lipid alterations.

Significant improvements in efficacy outcomes, including DAS-28 using erythrocyte sedimentation rate (ESR), tender and swollen joint count, Visual Analogue Scale (VAS), enthesitis and morning stiffness severity, were observed with apremilast at week 4. No changes on BMI were noticed. A significant reduction of intraarterial blood pressure was evidenced since the first 4 weeks. Serum levels of Apolipoprotein $A$ and $B$, insulin and HOMA-IR values were also significantly reduced after 24 weeks of treatment. Endothelial dysfunction was significantly restored shown by an increase of the peak flow and hyperaemia area and decreased adhesion molecules in serum. Levels of interleukins and adipokines were also modulated after apremilast treatment.

Conclusions:

- PsA is associated with an increase in inflammatory cytokines and adipokines, alongside with an endothelial dysfunction. These alterations are related to the disease activity and the presence of metabolic comorbidities such as insulin resistance or obesity, contributing to the burden of cardiovascular disease risk.

- Apremilast might reduce IR, inflammation, hypertension, lipids and endothelial dysfunction, parameters strongly involved in cardiovascular disease.

Acknowledgements: Supported by the Minister of Health (ISCIII, PI17/01316, RIER RD16/0012/0015) cofinanced with FEDER funds.

Disclosure of Interest: None declared

DOI: 10.1136/annrheumdis-2018-eular.6259

\section{SAT0332 A HIGH-DIMENSIONAL APPROACH TO DISSECTING THE ROLE OF THE TISSUE MICROENVIRONMENT IN SHAPING THE IMMUNE RESPONSE IN PSORIATIC ARTHRITIS}

J.H.S. Chan ${ }^{1}$, Y.Y. Leung ${ }^{2}$, W. Fong ${ }^{2}$, Y.W.K. Yeo ${ }^{2}$, B. Paleja ${ }^{1}$, L. Lai ${ }^{1}$, S. Saidin ${ }^{1}$, C. Chua ${ }^{1}$, S. Nur Hazirah ${ }^{1}$, S.L. Poh ${ }^{1}$, H.L.A. Low ${ }^{2}$, S. Albani ${ }^{1}{ }^{1}$ Translational Immunology Institute (TII)/Singapore Health Services Pte Ltd; ${ }^{2}$ Singapore General Hospital, Singapore, Singapore

Background: Psoriasis (Ps) currently inflicts $2 \%-3 \%$ of the population globally and one-third of patients have psoriatic arthritis (PsA). Up to $30 \%$ of PsA patients with active psoriasis (Ps) do not respond adequately to any treatment. Understanding the immune mechanisms contributing to the initiation and disease progression of PsA and Ps is crucial for devising novel therapeutic strategies.

Objectives: To address the current unmet clinical need and bridge the knowledge gap in the pathogenesis underlying PsA/Ps, we perform transcriptomic analyses of the skin microenvironment and deep immunophenotyping of immune cells from PsA patients with active disease. We hypothesise that the interaction between the tissue microenvironment and the peripheral immune system dictates the immune response that impacts upon the development and progression of $\mathrm{PsA} / \mathrm{Ps}$. This multi-dimensional strategy will also enable the distillation of immune cell subsets in the periphery that can potentiate pathogenic responses in the microenvironment.

Methods: Total RNA was extracted from skin punch biopsies of lesional and morphologically normal sites from 7 patients with active disease. RNASeq was performed to decipher the transcriptomes of the skin punch biopsies. Peripheral Blood Mononuclear Cells (PBMCs) from 17 PsA patients and 12 healthy donors were stimulated with PMA-lonomycin, stained with 37 phenotypic $T$ cells markers and interrogated with the CyTOF platform. Dimensional reduction and unsupervised clustering analyses were performed with Multi-dimensional Automated Reduction and Visualisation (MARVis).

Results: Transcriptomic analysis of skin punch biopsies of psoriatic and morphologically normal sites revealed a gene signature in lesional skin that promotes the infiltration of multiple immune cell subsets into the microenvironment. The expression of chemokine genes such as CXCL8, CCL4, and CCL20 suggests a role for the accumulation of neutrophils, monocytes, natural killer (NK) cells and lymphocytes in the establishment of a pro-inflammatory microenvironment that is perpetuated by the presence of TNF and IFN $\gamma$.

Examination of the immune landscapes of PsA patients highlights multiple perturbations in various immune cell subsets. Specifically, we observed declines in CD8 +CD161+TCRV $\alpha 7.2+$ Mucosal Associated Invariant $T$ (MAIT), CD4 +CD45RO+CXCR5+TFH as well as CD45RO+Tbet+IFN $\gamma+$ TNF $\alpha+$ IL17A +memory TH1 cells in PsA patients. This decline is potentially attributed to the trafficking of these immune cell subsets into the microenvironment in response to chemokine signals. Conversely, we observed enrichments of CD56 + Tbet
+ GranB +IFN $\gamma+\mathrm{NK}$ and activated CD4 +CD127+CCR7+CD69+effector T cells in PsA patients that can contribute to the pathogenic immune response.

Conclusions: Our multi-dimensional approach resolves the complex interactions between the tissue microenvironment and the peripheral immunome that shapes the immune response and dictates the cellular composition in lesional skin. These findings possess translational value and will facilitate the identification of novel immune therapeutic targets.

Disclosure of Interest: None declared

DOI: 10.1136/annrheumdis-2018-eular.6115

\section{SAT0333 DRUG SURVIVAL ON FIRST TNF INHIBITORS IN PATIENTS WITH PSORIATIC ARTHRITIS: COMPARISON ACROSS ETANERCEPT, ADALIMUMAB, GOLIMUMAB AND INFLIXIMAB}

C. García-Porrúa ${ }^{1}$, F. Maceiras-Pan² ${ }^{2}$ L. Fernandez-Dominguez ${ }^{3}$, J.A. MosqueraMartinez ${ }^{4}$, B. Correa-Rey ${ }^{5}$, M. Pombo-Suarez ${ }^{6}$, J. Pinto-Tasende ${ }^{7}$.

${ }^{1}$ Rheumatology, HU Lucus Augusti, Lugo; ${ }^{2}$ Rheumatology, CHU Vigo, Vigo;

${ }^{3}$ Rheumatology, CHU Ourense, Ourense; ${ }^{4}$ Rheumatology, CHU Pontevedra,

Pontevedra; ${ }^{5}$ heumatology, CHU Arquitecto Marcide-Naval, Ferrol;

${ }^{6}$ Rheumatology, CHU Santiago, Santiago de Compostela; ${ }^{7}$ Rheumatology, INIBICXXI A Coruña, A Coruña, Spain

Background: It is commonly accepted that additional methotrexate (MTX) does not increase efficacy for treatment with TNF in psoriatic arthritis (PsA) but concomitant MTX has been also associated with increased drug survival in register studies. However, the role of MTX comedication in PsA is still unclear.

Objectives: We aim was to evaluate TNF- $\alpha$ inhibitor (TNFi) persistence when used as first -line biologic therapy for the management of PsA naïve to biologic and determine features of patients as MTX comedication that are associated with TNFi persistence.

Methods: A ambispective longitudinal observational multi-centre cohort study was performed on all patients with PsA starting first TNFi therapy (etanercept adalimumab, golimumab and infliximab) between 01/JUN/2003 and 01/12/2015 Demographic and clinical data, concomitant treatment with MTX, were compared with TNFi persistence, using Kaplan-Meier survival and Cox regression analysis.

Results: Were included 468 patients starting treatment with etanercept (242), adalimumab (120), golimumab ${ }^{31}$ and infliximab (75); 235 receiving TNFi as monotherapy $(50.2 \%)$ and 233 receiving concomitant MTX (49.8\%). Mean age was $53.3 \pm 12.6$ years (men $53.3 \pm 12.9$ and women $53.2 \pm 12.3, p=0.928$ ) and $55.2 \%$ were men. Obesity $(\mathrm{BMI} \geq 30$ ) was similar in patients with or without persistence on treatment: 30.39 (13.32) vs 29.20 (5.44), $p=0.355)$. At 60 months of follow-up $50.6 \%$ of patients persisted with TNFi therapy (55.8\% with etanercept, $50.0 \%$ with adalimumab, $29.3 \%$ with infliximab and $67.7 \%$ with golimumab). Infliximab had the lowest retention rate $(p=0.006)$. Drug survival analyses had not differences between patients receiving co-medication or not $(p=0.849)$. In the Cox regression analysis lack of concomitant MTX and gender female were predictors of discontinuation of TNFi $(0.014)$

Conclusions: At 60 months infliximab had the lowest percentage of treatment continuation compared with etanercept, adalimumab and golimumab. Patients with PsA who are female and have not concomitant treatment with MTX could have lower TNFi persistence.

\section{REFERENCE:}

[1] Fagerli KM, Lie E, van der Heijde D, et al. The role of methotrexate comedication in TNF-inhibitor treatment in patients with psoriatic arthritis: results from 440 patients included in the NOR-DMARD study. Annals of the Rheumatic Diseases 2014;73:132-137

Acknowledgements: The authors are grateful for the support of the members of the Galician Society of Rheumatology (SOGARE)

Disclosure of Interest: None declared

DOI: 10.1136/annrheumdis-2018-eular.4951

\section{SAT0334 \\ CLINICAL AND SONOGRAPHIC ANALYSIS OF PSORIASIS PATIENTS WITHOUT MUSCULOSKELETAL COMPLAINTS. PREELIMINARY RESULTS OF A PROSPECTIVE STUDY: THE PRE-APS COHORT}

J. Ramírez García ${ }^{1}$, A. Cuervo ${ }^{1}$, M. Alsina ${ }^{2}$, R. Celis ${ }^{1}$, V. Ruiz-Esquide ${ }^{1}$, R. Castellanos ${ }^{1}$, M.V. Hernández ${ }^{1}$, R. Sanmarti ${ }^{1}$, J.D. Cañete ${ }^{1} .{ }^{1}$ Rheumatology; ${ }^{2}$ Dermatology, Hospital Clínic, Barcelona, Barcelona, Spain

Background: Early diagnosis in psoriatic arthritis (PsA) is mandatory in order to initiate early therapy and prevent disability. Around $20 \%$ of patients with Psoriasis (PsO) routinely visited in Dermatology departments have PsA previously undiagnosed. 